Revista Brasileira de Meteorologia, v. 30, n. 3, 319 - 326, 2015

http://dx.doi.org/10.1590/0102-778620130645

\title{
PREVISÃO DA UMIDADE RELATIVA DO AR DE BRASÍLIA POR MEIO DO MODELO BETA AUTORREGRESSIVO DE MÉDIAS MÓVEIS
}

\author{
DÉBORA MISSIO BAYER ${ }^{1}$, FÁBIO MARIANO BAYER ${ }^{2}$
}

\author{
${ }^{1}$ Universidade Federal de Santa Maria, Departamento de Engenharia Sanitária e Ambiental e Laboratório de \\ Ciências Espaciais de Santa Maria (LACESM), Santa Maria, RS, Brasil \\ ${ }^{2}$ Universidade Federal de Santa Maria, Departamento de Estatística e Laboratório de Ciências Espaciais de \\ Santa Maria (LACESM), Santa Maria, RS, Brasil
}

debora.bayer@ufsm.br, bayer@ufsm.br

Recebido Abril de 2013 - Aceito Dezembro de 2014

\begin{abstract}
RESUMO
O monitoramento da umidade relativa do ar (UR) tem grande importância no gerenciamento de recursos hídricos, na área agrícola, em estudos climáticos, assim como para a gestão da saúde pública. Este trabalho tem o objetivo de analisar, modelar e prever os valores mensais de UR da cidade de Brasília, Distrito Federal. Como a UR se dá em termos percentuais, ou seja, assume valores contínuos no intervalo $(0,1)$, os modelos de séries temporais tradicionais da classe ARIMA não são adequados. Com isso, a utilização do modelo beta autorregressivo de médias móveis ( $\beta$ ARMA) se faz necessária. Esse modelo para taxas e proporções, que assume distribuição beta para a variável de interesse, foi proposto recentemente por Rocha e Cribari-Neto. Para realização deste trabalho o modelo BARMA foi implementado em linguagem R. Sua aplicação aos dados de UR mostrou-se adequada, captando o comportamento da série e gerando previsões coerentes.
\end{abstract}

Palavras-chave: $\beta A R M A$; predição; séries temporais; umidade relativa do ar.

\begin{abstract}
RELATIVEAIRHUMIDITYFORECASTINGTHROUGHBETAAUTOREGRESSIVE MOVING AVERAGE MODEL IN BRASÍLIA

Relative humidity (RH) monitoring is of great importance for the management of water resources, agricultural field, climatic studies, as well as for public health management. The objective of this paper is to analyze, model, and forecast the monthly RH at Brasília city, Distrito Federal, Brazil. $\mathrm{RH}$ is given in percentage terms, i.e., assume continuous values in the interval $(0,1)$. In this situation the traditional time series models, like the ARIMA class, are not suitable. Thus, the use of beta autoregressive moving average models ( $\beta$ ARMA) is required. This model proposed by Rocha and Cribari-Neto was developed for rates and proportions variable with beta distribution. For this study, the $\beta A R M A$ model was implemented in $\mathrm{R}$ language. Its application to RH data was adequate, capturing the behavior of the RH series and producing accurate predictions.
\end{abstract}

Keywords: $\beta A R M A$; forecast; relative humidity; time series.

\section{INTRODUÇÃO}

Em algumas regiões do Brasil são registrados, todos os anos, períodos com baixos níveis de umidade relativa do ar (UR). Entender o comportamento desta variável no presente e determinar previsões fidedignas para o futuro podem auxiliar em medidas preventivas na área da saúde, assim como, em previsões climáticas e no gerenciamento dos recursos hídricos.
Tradicionalmente para modelagem e previsão de variáveis ao longo do tempo utilizam-se modelos autorregressivos integrados de médias móveis (ARIMA) (Box et al., 2008), como pode ser verificado em Bayer et al.(2012), Checi e Bayer (2012) e Prass et al. (2012). Especificamente com o objetivo de modelar e prever a variável UR, Attanayake (2008), Shiri et al. (2011), Syeda (2012) e Li et al. (2013) consideram essa classe de modelos. Contudo, os modelos ARIMA pressupõem normalidade da variável de interesse, o que pode se tornar 
restritivo ou inapropriado em vários estudos aplicados. Em especial, em situações que a variável resposta é limitada ao intervalo $(0,1)$, como ocorrem com taxas, proporções e variáveis percentuais, uma vez que o suporte da distribuição normal é toda a reta real e não apenas o intervalo unitário padrão $(0,1)$. Para situações como estas, Rocha e Cribari-Neto (2009) propõem o modelo beta autorregressivo de médias móveis (BARMA), assumindo que a variável aleatória de interesse $(y)$ possui distribuição beta. A densidade beta é bastante flexível, fato que torna o modelo $\beta$ ARMA muito útil para modelar a UR ao longo do tempo com o objetivo de traçar previsões.

Neste contexto, o presente trabalho aborda o problema da modelagem e da previsão da UR mínima mensal na cidade de Brasília. É apresentada uma breve discussão do problema climatológico, juntamente com a análise descritiva e caracterização dos dados. Para aplicação do modelo $\beta A R M A$ foi implementada pioneiramente uma função em linguagem $R$ (R Core Team, 2012). Essa função aborda aspectos de estimação pontual, testes de hipóteses, gráficos de qualidade do ajuste e previsões passos à frente.

\subsection{Por que estudar a umidade relativa do ar?}

A modelagem, o monitoramento e a previsão da UR são importantes em diversas áreas. Conhecer as características meteorológicas, dentre elas a umidade relativa do ar, auxilia a tomada de decisões como práticas de manejo, na área agrícola, na gestão dos recursos hídricos, na área de simulação hidrológica, ou de medidas preventivas, na área da saúde.

$\mathrm{Na}$ área agrícola, o conhecimento e/ou controle da UR tem por objetivo o manejo adequado (Sánchez-Blanco et al., 2004; Alnaizy e Simonet, 2012), o controle de ácaros (Boudreaux, 1958; Walzer et al., 2007), o controle de fungos (Costa et al., 2002; Silva et al., 2003; Del Ponte et al., 2004; Paul et al., 2004; Alves e Fernandes, 2006; Bosco, 2008), o controle de bactérias (Marcuzzo, 2008) e de insetos ( Roca e Larazzi, 1994), além da busca por um produto final com maior qualidade (Bilia et al., 1994; Costa et al., 2004; Dalmago et al., 2006; Goneli et al., 2007). Já na área de simulação hidrológica, a UR é importante na estimativa da evapotranspiração pela equação de Penman-Monteith, por exemplo, que é um dos modelos mais avançados utilizado em práticas hidrológicas, que representa o processo de evapotranspiração baseada no balanço de energia (Shuttleworth, 1992). Esta equação é muito utilizada em modelos de simulação hidrológica de base física, como o MGB-IPH (Modelos de Grandes Bacias) (Collischonn et al., 2007), o SWAT (Soil and Water Assessment Tool) (Arnold et al., 2012), o LARSIM (Large Area Runoff Simulation Model) (Ludwig e Bremicker, 2006), entre outros. Sendo assim, modelos que predizem adequadamente a UR podem se tornar ferramentas muito úteis para geração de dados de entrada (input) em modelos hidrológicos de base física.

Além da importância da UR na área agrícola, na área de simulação hidrológica e, consequentemente na engenharia de recursos hídricos, o seu monitoramento também se torna fundamental para a gestão da saúde pública. Valores muito baixos ou muito altos de UR podem estar relacionados a problemas de saúde (Sousa et al., 2007; Pinto et al., 2008; Silva-Junior et al., 2011). Segundo Barcellos et al. (2009), as características climáticas como temperatura, UR e precipitação, associadas às características físicas e químicas dos poluentes presentes na atmosfera, podem potencializar os efeitos causados por mudanças climáticas. Altas temperaturas e baixa umidade do ar favorecem o transporte de poluentes, que associados às condições climáticas podem afetar a saúde de populações, mesmo distantes das fontes geradoras de poluição. Isto pode aumentar os efeitos de doenças respiratórias e demais complicações associadas à baixa UR, como alergias, sangramentos nasais, ressecamento de pele e irritação nos olhos (Pinto et al., 2008). Já o aumento da temperatura e da UR podem estar relacionados à maior incidência de vetores de propagação de vírus e microrganismos causadores de doenças como meningite, dengue e pneumonia (Sousa et al., 2007).

Além das questões destacadas acima, a UR pode estar relacionada à ocorrência de incêndios em florestas e pastagens (Torres et al., 2009; Ribeiro et al., 2011), que agridem o ecossistema. Portanto, a modelagem, o monitoramento e as previsões adequadas da UR se fazem importantes nas mais diferentes áreas do conhecimento.

\section{MATERIAIS E MÉTODOS}

\subsection{O modelo}

Seja $y=\left(y_{1}, \ldots, y_{n}\right)^{T}$ um vetor com $n$ variáveis aleatórias, em que cada $y_{t}, t=1, \ldots, n$, tem distribuição condicional, dada pelo conjunto de informação prévia $F_{t-1}$, seguindo distribuição beta com parâmetro de média $\mu_{t}$ e de precisão $\phi$. Dessa forma, a densidade condicional de $y_{t}$ dado $\mathbb{F}_{t-1}$ é

$f\left(y_{t} \mid \mathbb{F}_{t-1}\right)=\frac{\Gamma(\phi)}{\Gamma\left(\mu_{t} \phi\right) \Gamma\left(\left(1-\mu_{t}\right) \phi\right)} y_{t}^{\mu_{t} \phi-1}\left(1-y_{t}\right)^{\left(1-\mu_{t}\right) \phi-1}$,

em que $0<y_{t}<1$ e $\Gamma$ (.) é função gama. Segue que a média condicional e a variância condicional de $y_{t}$, são respectivamente

$$
E\left(y_{t} \mid \mathbb{F}_{t-1}\right)=\mu_{t}, \quad \operatorname{var}\left(y_{t} \mid \mathbb{F}_{t-1}\right)=\frac{V\left(\mu_{t}\right)}{(1+\phi)},
$$

em que $V\left(\mu_{t}\right)=\mu_{t}\left(1-\mu_{t}\right)$ denota a função de variância. A densidade beta é bastante flexível para modelar dados climatológicos como a UR. Dependendo dos valores dos dois 
parâmetros que a indexam, a densidade assume formas bem variadas, acomodando distribuições simétricas, assimétricas, em formas de $\mathrm{J}$ e de $\mathrm{J}$ invertido. Isso é verificado pelas diversas densidades beta apresentadas na Figura 1.

Assumindo distribuição beta para a variável de interesse, o modelo $\beta$ ARMA $(p, q)$ pode ser escrito da seguinte forma (Rocha e Cribari-Neto, 2009):

$$
g\left(\mu_{t}\right)=\alpha+\sum_{i=1}^{p} \varphi_{i} g\left(y_{t-1}\right)+\sum_{j=1}^{q} \theta_{j} r_{t-1}
$$

em que $\alpha \in \mathbb{R}$ é uma constante, $\varphi^{\prime}$ 's e $\theta$ 's são, respectivamente, os parâmetros autorregressivos e de médias móveis, $r_{t}=y_{t}-\mu_{t}$ é o termo de erro de médias móveis e $g(\bullet)$ é uma função de ligação, assim como no modelo de regressão beta (Ferrari e Cribari-Neto, 2004). Neste trabalho foi considerada a função de ligação logit, i.e,

$$
\operatorname{logit}\left(\mu_{t}\right)=\log \left(\frac{\mu_{t}}{1-\mu_{t}}\right) \text {. }
$$

Os parâmetros do modelo são estimados via máxima verossimilhança, por meio da maximização do logaritmo da função de verossimilhança. A função de log-verossimilhança condicional é dada por:

$$
\ell=\ell(\theta ; y)=\sum_{t=m+1}^{n} \log f\left(y_{t} \mid \mathbb{F}_{t-1}\right)=\sum_{t=m+1}^{n} \ell_{t}\left(\mu_{t}, \phi\right),
$$

em que $\mathrm{m}=\max (p, q) \mathrm{e}$

$$
\begin{aligned}
& \ell_{t}\left(\mu_{t}, \phi\right)=\log \Gamma(\phi)-\log \Gamma\left(\mu_{t} \phi\right)-\log \Gamma\left(\left(1-\mu_{t}\right) \phi\right)+ \\
& \left(\mu_{t}-1\right) \log y_{t}+\left\{\left(1-\mu_{t}\right) \phi-1\right\} \log \left(1-y_{t}\right) .
\end{aligned}
$$

Para a maximização da função dada na Equação 1 se faz necessário o uso de algoritmos de otimização não linear. $\mathrm{Na}$ implementação computacional deste trabalho foi usado o algoritmo quasi-Newton BFGS (Press et al., 1992). Para maiores detalhes teóricos a respeito de inferências em grandes amostras e expressões matriciais para o vetor score e para a matriz de informação de Fisher, ver Rocha e Cribari-Neto (2009).

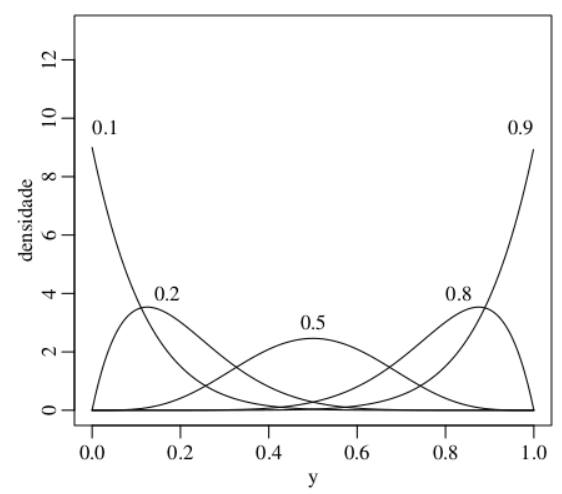

(a) $\phi=10$
Para a análise de diagnóstico de um modelo ajustado podem ser considerados diferentes resíduos. Neste trabalho foram considerados os resíduos padronizados, dados por:

$$
r_{t}^{p}=\frac{y_{t}-\hat{\mu}_{t}}{\sqrt{v \hat{a} r\left(y_{t}\right)}}=\frac{y_{t}-\hat{\mu}_{t}}{\sqrt{\frac{\sqrt{\mu_{t}\left(1-\mu_{t}\right)}}{(1+\hat{\phi})}}}
$$

Se o modelo estiver adequadamente ajustado é esperado que esses resíduos tenham média zero e estejam aleatoriamente distribuídos no intervalo $(-3,3)$.

\subsection{Caracterização da região e dos dados em estudo}

O Instituto Nacional de Pesquisas Meteorológicas (INMET) mantém diversas estações climatológicas no país, monitorando variáveis como: UR, precipitação, temperatura, velocidade e direção do vento, pressão atmosférica e insolação. Uma destas estações, cujos dados serão utilizados neste estudo, está localizada na cidade de Brasília, Distrito Federal, região Centro-Oeste do Brasil, aproximadamente a $15^{\circ} 48^{\prime}$ sul e a $48^{\circ}$ 55 ' oeste (conforme Figura 2).

Os valores que compõem a série de interesse correspondem a UR mínima registrada em cada mês, às 18 horas de cada dia (disponíveis em INMET (2013)). Assim, a série avaliada neste trabalho possui escala temporal mensal de janeiro de 2000 até fevereiro de 2013 . O período de janeiro de 2000 a junho de 2012 foi utilizado na análise e estudo da série temporal, enquanto o período de julho de 2012 a fevereiro de 2013 foi utilizado para verificação das previsões passos à frente.

A região de Brasília, de acordo com os dados disponíveis em INMET (2013), possui uma precipitação média anual de cerca de $1500 \mathrm{~mm}$, sendo que aproximadamente $1300 \mathrm{~mm}$ correspondem ao período chuvoso, de outubro a março, e apenas cerca de $200 \mathrm{~mm}$ ao período seco, de abril a setembro. A sazonalidade da precipitação média mensal pode ser vista na Figura 3. A temperatura máxima registrada no período estudado

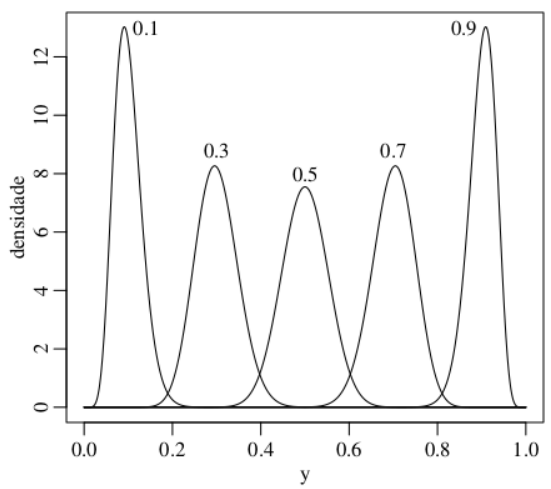

(b) $\phi=90$

Figura 1: Densidades beta para diferentes valores de $\mu$ (indicados no gráfico), com $\phi=10$ (a) e $\phi=90$ (b). 

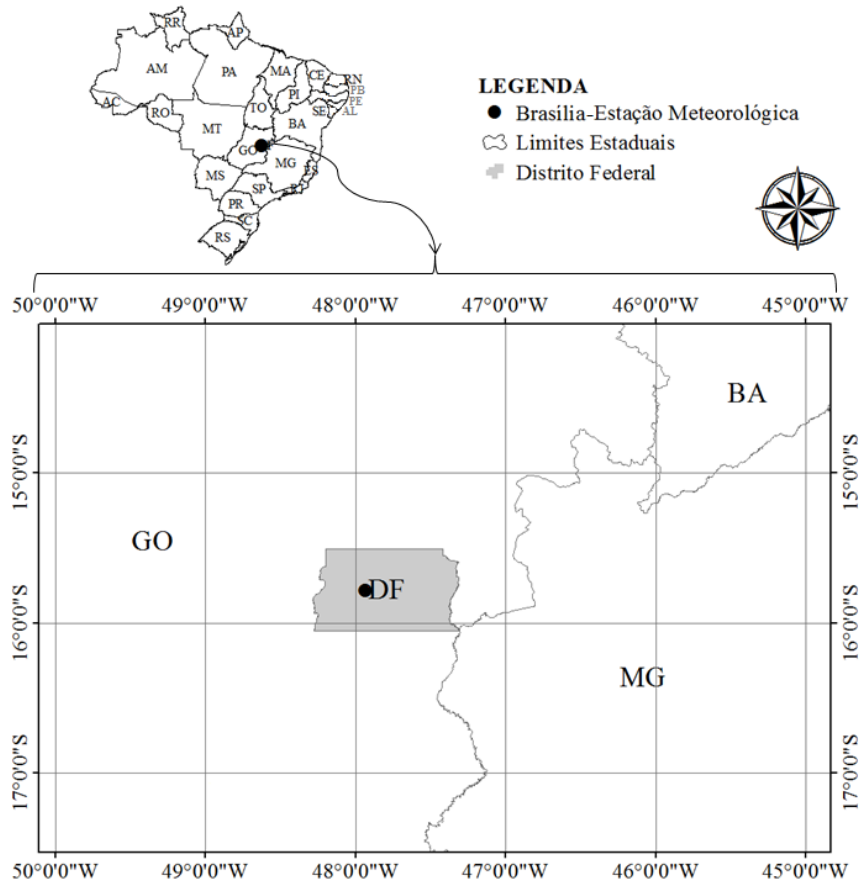

Figura 2: Localização da região em estudo.

foi de $35,8^{\circ}$, em outubro de 2008 , e a mínima de $8.2^{\circ}$, em julho de 2000 .

\section{RESULTADOS E DISCUSSÕES}

\subsection{Análise descritiva dos dados}

Uma primeira análise descritiva dos dados estudados pode ser feita pela simples inspeção visual da série temporal, conforme a Figura 4, assim como pelo cálculo de algumas medidas descritivas simples, apresentadas na Tabela 1. No período analisado o valor médio da variável UR mínima

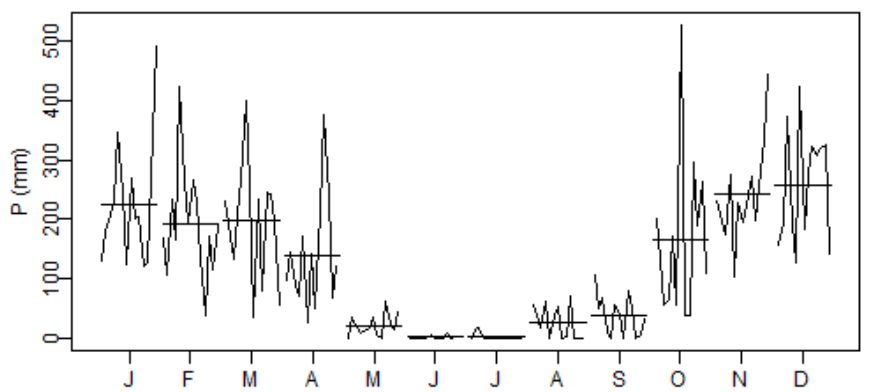

Figura 3: Subséries de precipitações mensais em Brasília, caracterizando a sua sazonalidade. mensal é de $29,4 \%$, com coeficiente de variação de $32,3 \%$. A variabilidade da UR pode ser verificada na Figura 4, em que o valor mínimo registrado foi de $10 \%$ no mês de setembro de 2011 e o valor máximo foi de $52 \%$ em março de 2005 . As linhas horizontais tracejadas nas Figuras 4 e 5 correspondem aos limites máximos do intervalo de atenção (entre 20 e $30 \%$ ), de alerta (entre 12 e $20 \%$ ) e de emergência (abaixo de $12 \%$ ) associados a medidas de prevenção à problemas de saúde devido à baixa UR (Pinto et al., 2008).

A análise gráfica evidencia a sazonalidade da UR, principalmente a Figura 5. Há uma grande variabilidade intra-anual, alcançando em média os menores valores no mês de agosto e os maiores valores no mês de março. A umidade do ar está associada ao movimento das massas de ar e à transferência de água do estado líquido para o gasoso, por meio da evapotranspiração das plantas e das superfícies evaporativas.

Como comentado anteriormente, nesta região o período de chuvas inicia-se em outubro, estendendo-se até meados do mês de abril, quando a intensidade e frequência das chuvas

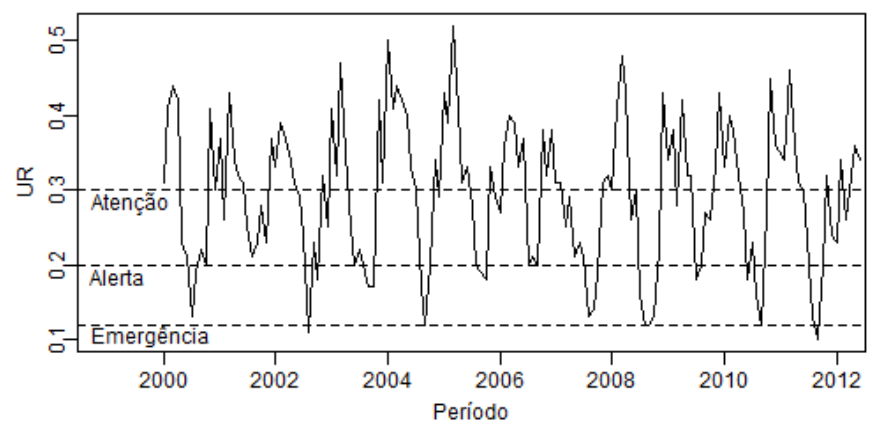

Figura 4: Série temporal de mínimas mensais da umidade relativa do ar em Brasília. Limites de Atenção, Alerta e Emergência indicados por Pinto et al. (2008).

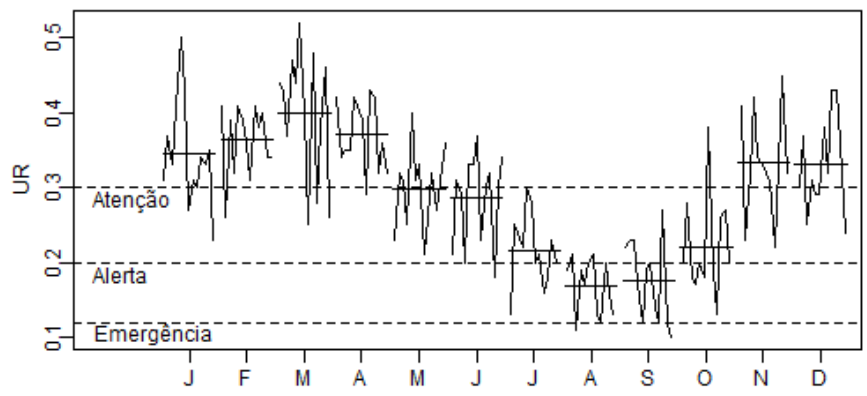

Figura 5: Subséries de mínimas mensais da umidade relativa do ar em Brasília caracterizando a sua sazonalidade. Limites de Atenção, Alerta e Emergência indicados por Pinto et al. (2008).

Tabela 1: Medidas descritivas da variável UR mínima mensal.

\begin{tabular}{cccccc}
\hline Média & Mediana & Desvio-Padrão & Coeficiente de Variação & Mínimo & Máximo \\
0,294 & 0,310 & 0,095 & 32,313 & 0,100 & 0,520 \\
\hline
\end{tabular}


começam a reduzir, atingindo valores mínimos de maio a setembro. No período mais chuvoso é esperado que a UR seja maior, uma vez que a umidade do ar origina a chuva, ao mesmo tempo que a atmosfera passa a ser reabastecida de vapor de água pela própria chuva que passa a evaporar. Após cessar o período de chuvas, a UR permanece elevada por mais alguns meses, o que pode ser explicado pelo volume de água armazenado no sistema durante o período chuvoso, e diminui gradativamente até atingir valores mínimos nos meses de agosto e de setembro, voltando a crescer quando chega novamente a estação chuvosa.

\subsection{Aplicação do modelo ßARMA}

Parte fundamental no ajuste dos modelos de séries temporais do tipo ARIMA ou $\beta$ ARMA é a determinação das ordens $p$ e $q$ dos modelos. Neste sentido, para a seleção do modelo foi considerada uma busca computacionalmente exaustiva com o objetivo de minimizar o critério de informação de Akaike (AIC) (Akaike, 1973). O espaço de busca foi restringido aos modelos com ordens menores ou iguais a 4 , isto é, considerou-se $0 \leq p \leq 4$ e $0 \leq q \leq 4$. Como resultado desta busca, o modelo $\beta$ ARMA $(4,4)$ foi o que obteve menor AIC. Contudo, os coeficientes relativos a $\varphi_{2}, \varphi_{3}$ e $\theta_{2}$ não se

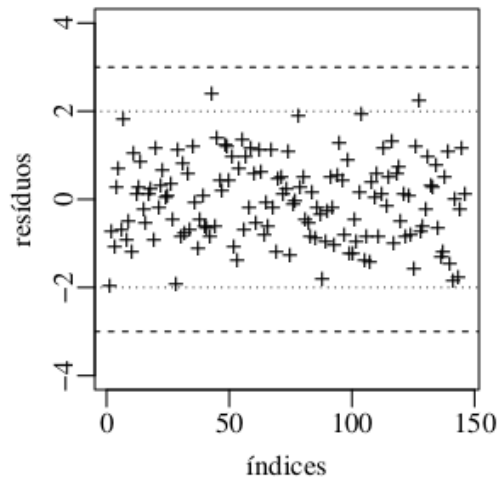

(a) Resíduo vs Índices

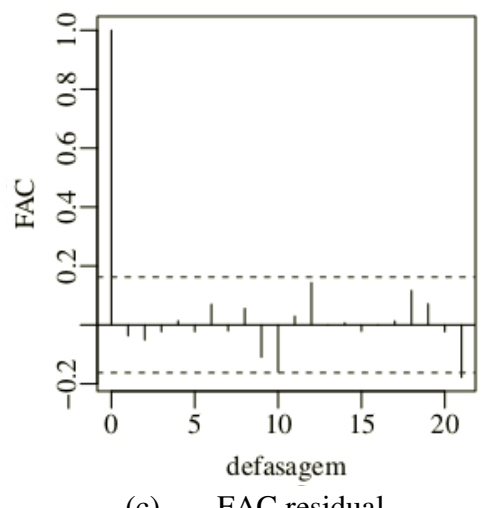

(c) FAC residual mostraram significativos a níveis nominais usuais, ou seja, as componentes autorregressivas de ordens 2 e 3 e a componente de médias móveis de ordem 2 não devem estar presentes no modelo. O modelo resultante está apresentado na Tabela 2.

A Figura 6 apresenta a análise de diagnóstico do modelo selecionado. Os gráficos evidenciam um bom ajuste do modelo, uma vez que os correlogramas residuais, Figuras $6 c$ e 6d, não apresentam auto-correlação significativamente diferente de zero e os resíduos padronizados distribuem-se normalmente ao redor de zero e dentro do intervalo de -3 a 3 , conforme Figura 6 a e 6b. A análise de diagnóstico valida o modelo selecionado para traçar previsões. A Figura 7 apresenta graficamente os valores reais observados e os valores preditos pelo modelo no intervalo

Tabela 2: Modelo $\beta$ ARMA ajustado aos dados de umidade relativa do ar.

\begin{tabular}{|c|c|c|c|c|}
\hline & Estimativa & Erro Padrão & Valor z & $\operatorname{Pr}(>|z|)$ \\
\hline$\alpha$ & $-0,5597$ & 0,0882 & 6,3437 & 0,0000 \\
\hline$\varphi_{1}$ & 0,7942 & 0,0649 & 12,2451 & 0,0000 \\
\hline$\varphi_{4}$ & 0,4254 & 0,0643 & 6,6131 & 0,0000 \\
\hline$\theta_{1}$ & $-2,1294$ & 0,5082 & 4,1901 & 0,0000 \\
\hline$\theta_{3}$ & $-1,4953$ & 0,3854 & 3,8794 & 0,0001 \\
\hline$\theta_{4}$ & 2,4789 & 0,4953 & 50,0049 & 0,0000 \\
\hline$\phi$ & 38,2818 & 4,4347 & 8,6324 & 0,0000 \\
\hline \multicolumn{5}{|c|}{ Log-verossimilhança maximizada:191,3475 } \\
\hline & \multicolumn{2}{|c|}{ AIC: $-368,69$} & $:-347,62$ & \\
\hline
\end{tabular}

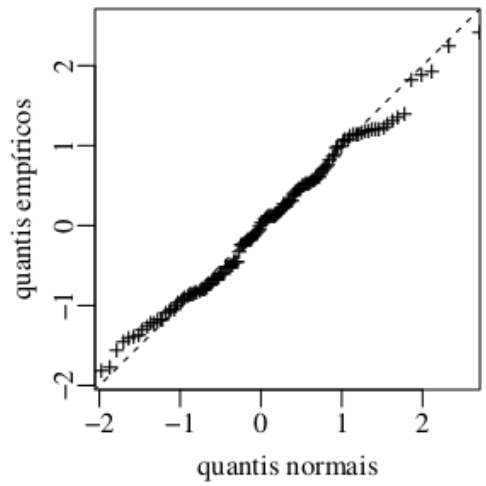

(b) Q-Q plot

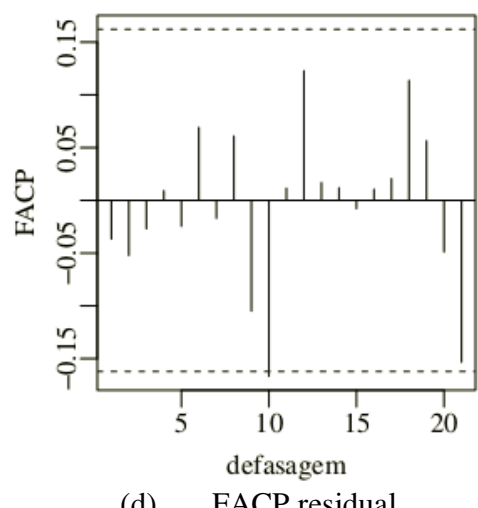

(d) FACP residual

Figura 6: Análise de diagnóstico do modelo ajustado. 
de observações. O gráfico evidencia a boa qualidade de ajuste, mostrando valores reais e previstos muito próximos.

Destaca-se, na utilização do modelo $\beta$ ARMA para previsão da variável UR, o fato do suporte da distribuição beta ser restrito ao intervalo de possíveis valores da UR, ou seja, o intervalo $(0,1)$. Desta maneira, valores preditos menores que $0 \%$ ou maiores $100 \%$ de UR não são possíveis de ocorrer. Por outro lado, ao se considerar os tradicionais modelos da classe ARIMA, utilizados para modelagem UR por Attanayake (2008), Shiri et al. (2011), Syeda (2012) e Li et al. (2013), podem ocorrer valores irreais de UR, acima de $100 \%$ ou abaixo de $0 \%$, uma vez que o suporte da distribuição normal é toda a reta real.

As previsões passos à frente, assim como os valores reais para este período, são apresentadas na Figura 8. O mês de fevereiro de 2013 apresentou o maior valor previsto para a UR, de $32,7 \%$, enquanto o menor foi previsto para agosto de 2012, de $23,5 \%$. Esses valores são coerentes com o histórico da série real observada, com valores máximos e mínimos correspondendo aos períodos úmido e seco, respectivamente. A previsão para o mês de novembro de 2012 foi de $26,5 \%$, enquanto que o valor real registrado foi de $44,0 \%$. A diferença entre o valor previsto e o real pode ser explicada pela precipitação atípica registrada no mês de novembro na região, cerca de $444 \mathrm{~mm}$, enquanto a média de precipitação para o mês de novembro no período de ajuste do modelo foi de cerca de $224 \mathrm{~mm}$. Cabe destacar que se fossem utilizados os modelos ARIMA, que assumem distribuição normal para a variável de interesse, como em Bayer et al.(2012), Checi e Bayer (2012) e Prass et al. (2012), poderse-ia ter valores previstos para UR fora do intervalo $(0,1)$, o que não é razoável para previsões de UR. Já com o uso do modelo $\beta A R M A$ garante-se que todas as previsões estarão no intervalo $(0,1)$, pois esse é o suporte da distribuição beta, assumida para os dados de interesse nesse modelo.

\section{CONCLUSÕES}

O trabalho apresentou uma aplicação do modelo $\beta A R M A$ para previsão da umidade relativa do ar na cidade de Brasília, DF, Brasil. Trata-se da primeira aplicação, dentro do conhecimento

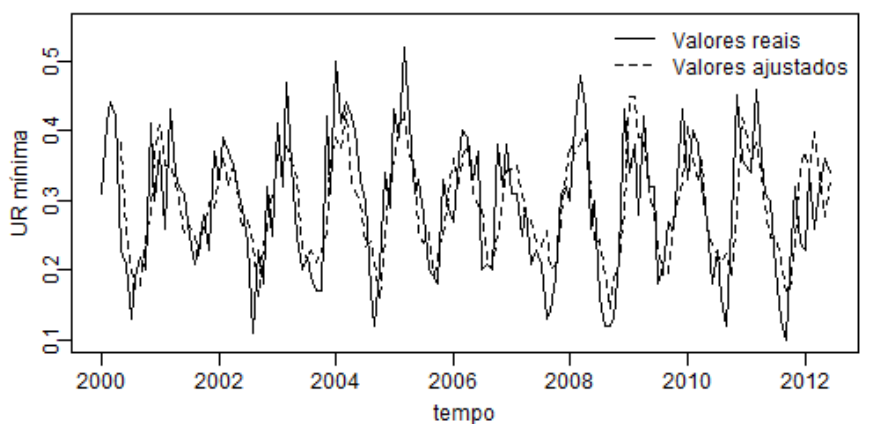

Figura 7: Valores reais observados e valores ajustados pelo modelo.

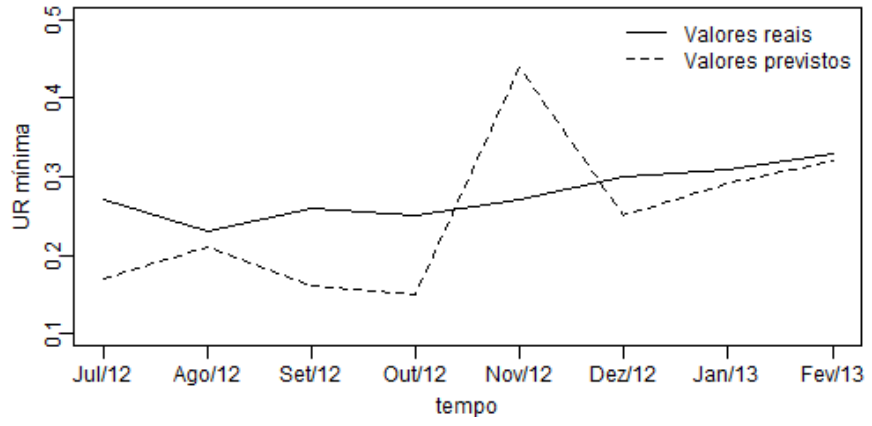

Figura 8: Valores reais e previsões passos à frente.

dos autores, deste modelo de séries temporais específico para modelagem de variáveis do tipo taxa e proporção. Para o desenvolvimento do trabalho foi necessária a implementação do modelo em uma linguagem de programação. Optou-se pelo desenvolvimento de uma função no software $R$, que envolve aspectos de estimação pontual, testes de hipóteses, gráficos de qualidade do ajuste e previsões passos à frente. Futuramente esta função será disponibilizada na rede e poderá se tornar um pacote do R.

O modelo se mostrou bastante útil para a modelagem e previsão da UR, captando as características da série e provendo previsões coerentes. A previsão de variáveis meteorológicas é importante para a gestão dos recursos hídricos e para a área agrícola, podendo servir como input para modelos físicos ou mesmo para caracterização direta do clima de determinadas regiões. Além disso, as previsões podem auxiliar na tomada de decisões para controle de problemas relacionados à saúde.

\section{AGRADECIMENTOS}

Os autores agradecem à Fundação de Amparo à Pesquisa do Estado do Rio Grande do Sul (FAPERGS) e ao Conselho Nacional de Desenvolvimento Científico e Tecnológico (CNPq) pelo apoio à pesquisa.

\section{REFERÊNCIAS BIBLIOGRÁFICAS}

AKAIKE, H. A new look at the statistical model identification. IEEE Transactions on Automatic Control, v.19, p.716723, 1973.

ALNAIZY, R.; SIMONET, D. Analysis of water evaporation and drift losses during irrigation in semi-arid areas of Sharjah (UAE) and Riyadh (KSA). Natural Resources Research, v. 21, n. 2, p. 285-299, 2012.

ALVES K. J. P.; FERNANDES, J.M.C. Influência da temperatura e da umidade relativa do ar na esporulação de Magnaporthe grisea em Trigo. Fitopatologia Brasileira, v. 31, p. 579-584, 2006. 
ARNOLD, J.G. et al. Soil \& Water Assessment Tools. Input/output documentation. Texas Water Resources Institute. 2012. 650 p.

ATTANAYAKE, A. M. C. H. A univariate Box-Jenkins model to predict relative humidity levels in Puttalam at night. In PROCEEDINGS OF THE ANNUAL RESEARCH SYMPOSIUM. Anais...Sri Lanka:Faculty of Graduate Studies, University of Kelaniya Kelaniya, 2008, p. 138.

BARCELLOS, C. et al. Mudanças climáticas e ambientais e as doenças infecciosas: cenários e incertezas para o Brasil. Epidemiologia e Serviços de Saúde. v. 18, n. 3, p. 285 304, 2009.

BAYER, D. M., CASTRO, N. M., BAYER, F. M. Modelagem e previsão de vazões médias mensais do Rio Potiribu utilizando modelos de séries temporais. Revista Brasileira de Recursos Hídricos. v. 17, p.229-239, 2012.

BILIA, D.A.C.; FANCELLI, A.L., MARCOS FILHO, J. Comportamento de sementes de milho híbrido durante o armazenamento sob condições variáveis de temperatura e umidade relativa do ar. Scientia Agricola, v. 51, n. 1, p. 153-157, 1994.

BOSCO, L. C. Sistemas de previsão de ocorrência de requeima em clones de batata suscetíveis e resistentes. 2008, 114 f. Dissertação (Mestrado em Agronomia) Universidade Federal de Santa Maria, Santa Maria, 2008.

BOUDREAUX, H. B. The effect of relative humidity on egg-laying, hatching, and survival in various spider mites. Journal of Insect Physiology, v. 2, n. 1, p. 65-72, 1958.

BOX, G.; JENKINS, G.;REINSEL, G. Time Series Analysis: forecasting and control. 4 ed.. John Wiley \& Sons, 2008, $784 \mathrm{p}$.

CHECI, L.;BAYER, F. M. Modelos univariados de séries temporais para previsão das temperaturas médias mensais de Erechim, RS. Revista Brasileira de Engenharia Agrícola e Ambiental, v. 16, n. 12, p. 1321-1329, 2012.

COLLISCHONN W et al.. The MGB-IPH model for largescale rainfall-runoff modelling. Hydrological Sciences Journal v. 52, p. 878-895, 2007.

COSTA, E.; LEAL, P.A.M.;DO CARMO JÚNIOR, R.R. Modelo de simulação da temperatura e umidade relativa do ar no interior de estufa plástica. Engenharia Agrícola, v. 24, n. 1, p.57-67, 2004.

COSTA, R.V. et al. Previsão da requeima da batateira. Fitopatologia Brasileira, v. 27, p. 349-354, 2002.

DALMAGO, G.A. et al. Evapotranspiração máxima da cultura de pimentão em estufa plástica em função da radiação solar, da temperatura, da umidade relativa e do déficit de saturação do ar. Ciência Rural, v. 36, n. 3, p.7 85-792, 2006.

DEL PONTE, E.M. et al. Giberela do trigo - aspectos epidemiológicos e modelos de previsão. Fitopatologia Brasileira., v. 29, p. 587-605, 2004.
FERRARI, S. L. P., CRIBARI-NETO, F.. Beta regression for modelling rates and proportions. Journal of Appied Statistics v. 31, n. 7, p. 799-815, 2004.

GONELI, A.L.D.; CORRÊA, P.C.; RESENDE, O.; REIS NETO, S.A. Estudo da difusão de umidade em grãos de trigo durante a secagem. Ciência e Tecnologia de Alimentos, v. 27, n. 1, p. 135-140, 2007.

INMET. Dados da rede do Instituto Nacional de Meteorologia. Disponível em: <http://www.inmet.gov.br/projetos/rede/ pesquisa/>. Acesso em: 08 out 2012 e 01 abr 2013.

LI et al. Hadoop-based ARIMA algorithm and its application in weather forecast. International Journal of Database Theory and Application, v. 6, n. 5, p. 119-132, 2013.

LUDWIG, K.; BREMICKER, M.. The water balance model LARSIM: design, content and applications. Freiburger Schriften zur Hydrologie 22. Institut für Hydrologie Universität Freiburg, Germany. 2006.

MARCUZZO, L.L. Epidemiologia e previsão da mancha bacteriana (Xanthomonas spp.) do tomateiro. 2008, $81 \mathrm{f}$. Tese (Doutorado em Agronomia) - Faculdade de Agronomia e Medicina Veterinária da Universidade de Passo Fundo, Passo Fundo, 2008.

PAUL P. A. et al. Epidemiologia comparativa da pinta preta do tomateiro sob quatro regimes de pulverização. Fitopatologia Brasileira, v. 29, p. 475-479, 2004.

PINTO, H. S., ZULLO-JR., J., AVILA, A. M. H.. Umidade do ar: saúde no inverno. Setembro de 2008. Disponível em: $<$ http://www.cpa.unicamp.br/artigos-especiais/umidadedo-ar-saude-no-inverno.html> . Acesso em: 09 out 2012.

PRASS, T. S., et al. Comparison of forecasts of mean monthly water level in the Paraguay River, Brazil, from two fractionally differenced models. Water Resources Research, v.48, n.5, p.W05502. 2012.

PRESS, W. et al.. Numerical recipes in C: The art of scientific computing. $2^{\mathrm{a}}$ ed. Cambridge University Press. 1992. 1018p. Disponível em: <http://astronu.jinr.ru/wiki/upload/d/ d6/NumericalRecipesinC.pdf $>$

R CORE TEAM, R: A language and environment for statistical computing. R Foundation for Statistical Computing, Vienna, Austria, ISBN 3-900051-07-0. 2012. Disponível em: < http://www.R-project.org/ >

RIBEIRO, L., et al. Análise do perigo de incêndios florestais em um município da amazônia Mato-Grossense, Brasil. Floresta, v. 41, n. 2, p.257-270, 2011.

ROCA, M.J.; LAZZARI,C.R. Effects of relative humidity on the haematophagous bug Triatoma infestans : hygropreference and eclosion success. Journal of Insect Physiology, v. 40, n. 10, p. 901-907, 1994.

ROCHA, A.; CRIBARI-NETO, F. Beta autoregressive moving average models. Test, v.18, n.3, p. 529-545, 2009. 
SÁNCHEZ-BLANC et al. Effects of irrigation and air humidity preconditioning on water relations, growth and survival of Rosmarinus officinalis plants during and after transplanting. Journal of Plant Physiology, v. 161, p. 1133-1142, 2004.

SHIRI et al. The investigation and forecasting of relative humidity variation of Pars Abad-e-Moghan, North-West of Iron, by ARIMA model. Research Journal of Applied. Sciences, v. 6, n. 2, p. 81-87, 2011.

SILVA, M.D.D., ALFENAS, A.C., MAFFIA, L.A. \& ZAUZA, E.A.V. Germinação de conídios de Sphaerotheca pannosa obtidos de eucalipto. Fitopatologia Brasileira, v. 28, p. 674-681, 2003.

SILVA-JUNIOR, J. L. R., et al. Efeito da sazonalidade climática na ocorrência de sintomas respiratórios em uma cidade de clima tropical. Jornal Brasileiro de Pneumologia, v. 37, n.6, p.759-767, 2011.

SOUSA, N.M.N.; DANTAS, R.T.;LIMEIRA, R.C. Influência de variáveis meteorológicas sobre a incidência do dengue, meningite e pneumonia em João Pessoa-PB. Revista Brasileira de Meteorologia, v.22, n.2, p. 183-192, 2007.
SHUTTLEWORTH, W. J.. Evaporation In: MAIDMENT, D.R. (Org.). Handbook of Hydrology. McGraw-Hill. 1992. cap. 4.

SYEDA, J.A. Variability analysis and forecasting of relative humidity in Bangladesh. Journal of Environmental Science and Natural Resources, v.5, n. 2, p. 137-147, 2012. TORRES, F.T.P. et al. Relações entre incêndios em vegetação e elementos meteorológicos na cidade de Juiz de Fora, MG. Revista Brasileira de Meteorologia, v.24, n.4, p. 379-389, 2009.

WALZER, A. et al. Intraspecific variation in humidity susceptibility of the predatory mite Neoseiulus californicus: Survival, development and reproduction. Biological Control, v. 41, p. 42-52, 2007. 